\title{
WEAKLY NONLINEAR AND NUMERICAL ANALYSES OF DYNAMICS IN A SOLID COMBUSTION MODEL*
}

\author{
L. K. GROSS ${ }^{\dagger}$ AND J. YU
}

\begin{abstract}
This paper contains qualitative and quantitative comparisons between a weakly nonlinear analysis and direct numerical simulations of a free-boundary problem. The former involves modulating the most linearly unstable mode, taking a small perturbation of the neutrally stable value $\nu_{c}$ of a parameter $\nu$ related to the activation energy. Analogously, we perform the direct numerical computations near the marginally unstable value, namely, $\nu=\nu_{c}-\epsilon^{2}$, where $\epsilon$ is rather small.

We delineate the role of a different parameter $\sigma$ (related to the Arrhenius kinetics) in the combustion dynamics when $\nu=\nu_{c}-\epsilon^{2}$. In particular, the numerics show that varying $\sigma$ produces a period-doubling scenario when $\epsilon$ lies approximately between 0.08 and 0.12 . We describe the $\sigma$ intervals within which complex dynamics occur for various values of $\epsilon$ and for $\nu$ fixed at $\nu_{c}-\epsilon^{2}$. When $\epsilon$ drops to approximately 0.06 , the asymptotic and numerical solutions agree well for all physical values of $\sigma$.
\end{abstract}

Key words. free-boundary problems, condensed-phase combustion, weak instability, asymptotic expansions, Crank-Nicolson method, Fourier transforms

AMS subject classifications. 35R35, 80A25, 80M35, 80M25, 42A38

DOI. $10.1137 /$ S0036139904439508

1. Introduction. In this article, we study the nonuniform dynamics of front propagation in a free-boundary model of solid combustion, through both weakly nonlinear analysis and direct simulations. We do the first quantitative comparison of the two methods.

The asymptotic technique applies to the weakly unstable setting. In particular, we fix the bifurcation parameter $\nu$ related to the activation energy to within a rather small number $\epsilon^{2}$ of the neutrally stable value $\nu_{c}$. By solving numerically in the same regime, we closely investigate the role of a parameter $\sigma$ associated with the Arrhenius kinetics. In particular, period-doubling and eventual chaos develop as the kinetics parameter $\sigma$ decreases (and the bifurcation parameter $\nu$ remains at a deviation of $\epsilon^{2}$ from its critical value).

Weakly nonlinear analysis involves modulating the most linearly unstable mode. Within quite a small neighborhood of the neutral stability boundary, Fourier spectra of the numerical quasi-steady-state solutions indicate a regime in which a single mode dominates, as well as complex regimes of front propagation.

As the bifurcation parameter $\nu$ approaches ever closer to the neutrally stable value, the range of the parameter $\sigma$ for which period-doubling and other strongly nonlinear dynamics occur shrinks. Sufficiently near the stability threshold $(\epsilon$ approximately 0.06), numerical solutions for all values of $\sigma$ agree closely with the weakly

* Received by the editors January 6, 2004; accepted for publication (in revised form) November 3, 2004; published electronically July 13, 2005.

http://www.siam.org/journals/siap/65-5/43950.html

${ }^{\dagger}$ Department of Theoretical and Applied Mathematics, The University of Akron, Akron, OH 44325-4002 (gross@math.uakron.edu). The research of this author was supported by the National Science Foundation under grant DMS-0074965, the Ohio Board of Regents (Research Challenge Grant), and the Buchtel College of Arts and Sciences at The University of Akron (matching grant).

${ }^{\ddagger}$ Department of Mathematics and Statistics, The University of Vermont, Burlington, VT (jun.yu@ uvm.edu). 
nonlinear solutions. By varying $\epsilon$, we quantify the domain of applicability of the weakly nonlinear analysis.

The problem under consideration models, for example, solid combustion, in which a chemical reaction converts a solid fuel directly into solid products with no intermediate gas phase formation. For instance, in self-propagating high-temperature synthesis (SHS), a flame wave advancing through powdered ingredients leaves high-quality ceramic materials or metallic alloys in its wake. (See, for instance, $[15,17,20]$.)

The propagation results from the interplay between heat generation and heat diffusion in the medium. A balance exists between the two in some parametric regimes, producing a constant burning rate. In other cases, competition between the reaction and the diffusion results in a wide variety of nonuniform behaviors, some leading to chaos.

Shkadinsky, Khaikin, and Merzhanov [18] predicted the simplest oscillatory regimes through numerical simulation on reaction-diffusion partial differential equations (PDEs). The system contains Arrhenius-kinetics terms that account for chemical conversion throughout the spatial domain.

Various works have explored numerically the dynamics of models that employ approximations to the Arrhenius kinetics. For instance, in [1], Arrhenius kinetics with a cutoff was used to observe chaotic pulsations, following a number of perioddoubling bifurcations.

Other approximations exploit the narrowness of the reaction zone. A point-source model has an exact traveling-wave solution and is more amenable to analysis than one with the full Arrhenius kinetics. Matkowsky and Sivashinsky [14] studied a concentrated-kinetics model in the case of large activation energy. The $\delta$-function kinetics follow from an analysis similar to that of [19].

This free-interface problem has been studied numerically in [4]. For a sufficiently large activation energy, the work showed transitions to chaos via a period-doubling solution and highly irregular relaxational oscillations. The authors attributed a lack of sequential secondary bifurcations to the difference between the point-source and distributed-kinetics models (as in [1]). Later, however, in [9], the entire spectrum of behavior was observed for the free-interface model, as previously had been seen for distributed kinetics.

In [9], the authors performed numerical computations on a second model of solid combustion as well. They motivate it by noting that both the reaction-diffusion model as in [18] and the free-interface model in [14] assume a constant value of thermal diffusivity. However, some problems manifest a clear dependence of this parameter on degree of conversion. In fact, when the burnt product is a foam-like substance, heat diffusion in the product region is negligible. For such cases, they consider a model that includes the heat equation on a semi-infinite domain ahead of the reaction and a nonlinear kinetic condition imposed on the moving boundary. The present paper uses this free-boundary problem.

Note that both the free-interface (two-sided) model and the free-boundary (onesided) model stem from reaction-diffusion PDEs with full Arrhenius kinetics. To emphasize, the one-sided model is not an adaptation of the two-sided model; rather each of them is a viable derivative of the reaction-diffusion model. The two-sided model assumes a single constant conductivity throughout the reactant and product zones. The one-sided model assumes zero conductivity in the burned region. In some cases the first approximation is more appropriate, in others the second.

Belyaev and Komkova discovered a pulsating regime in the burning of a chrome- 
magnesium thermite in 1950 [2]. A planar front may have oscillated with a constant frequency in their experiments, but they did not observe the process in detail. Later Merzhanov, Filonenko, and Borovinskaya [16] observed experimentally both the periodic propagation of a flat front in SHS as well as spinning waves, showing a fuller understanding of the behaviors. All the models discussed in this literature review exhibit the same spectrum of dynamics as experiments. Specifically, we refer to computed solutions of (i) the reaction-diffusion system governed by the full Arrhenius kinetics (e.g., [5]), (ii) the reaction-diffusion system with Arrhenius kinetics with a cutoff (e.g., [1]), and models that use point-source kinetics like (iii) the free-interface ("two-sided") model with constant heat diffusivity (e.g., [9]), as well as (iv) the freeboundary ("one-sided") model, in which heat transfer behind the flame front (in the burned matter) is qualitatively unimportant (e.g., [9]).

Simulations on all these models show the same dynamical behaviors as one pushes the bifurcation parameters deeper into the instability regions. In particular, numerical simulations and analysis in [9] show that dynamics of the two-sided and one-sided problems agree extremely closely.

In the present work, we fix the bifurcation parameter $\nu$ within $\epsilon^{2}$ of the neutrally stable value and vary the kinetics parameter $\sigma$, rather than exploring regimes more and more strongly unstable in $\nu$. In addition, we vary $\epsilon$, thereby also changing $\nu$, and study the impact on the dynamics with respect to the kinetics parameter $\sigma$. We will point out the agreement with dynamical scenarios described in previous studies, which use a variety of models.

The stability thresholds for uniformly propagating fronts generally differ for all of the different kinetics mentioned, however. Distributed kinetics have only the numerical approximate bifurcation values. Intricate bifurcation analyses $[13,10]$ of instabilities for the point-source models have also classified the interactions of clockwise and counterclockwise spinning waves on the surface of a cylinder. Margolis's review paper [13] includes a thorough discussion of resonance phenomena, treating sample radii that yield close, as well as equal, eigenvalues. Also, Booty, Margolis, and Matkowsky [3] predicted cascades of bifurcations from a double eigenvalue of a linearized model of condensed-phase combustion in a long cylindrical sample. They show that the inclusion of melting in the model makes the neutral-stability threshold more accessible. A bifurcation parameter $\nu$ in the present work is restricted to a smaller neighborhood of the value corresponding to a single neutrally stable eigenvalue. A different parameter $\sigma$ is varied to produce period-doubling behaviors numerically.

Combustion in two dimensions can be described by a one-dimensional model when the only unstable mode corresponds to the dynamics with no spatial variation in the transverse direction. For example, the linear stability analysis in [12] shows that for a free-boundary model, a flat front dominates the behavior for the case of a sufficiently narrow strip of material with insulated edges.

In particular, to satisfy the boundary conditions, the wave numbers are integer multiples of $\pi / a$, where $a$ is the strip width [12]. If $a<\pi$, all modes are stable for $\nu>1 / 3$. Exactly one mode (the zeroth mode) loses stability at $\nu_{c}=1 / 3$. The zero mode corresponds to the dynamics with no spatial variation in the transverse direction (i.e., to the one-dimensional case). If, on the other hand, $\pi<a<2 \pi$, then, as we decrease $\nu$, the first mode $\pi / a$ loses stability prior to the flat mode, namely, at a value of $\nu>1 / 3$. In both cases $(a<\pi$ and $\pi<a<2 \pi)$ the weakly nonlinear analysis shows that the evolution is governed by a complex Landau-Stuart ordinary differential equation [11]. (See what follows for the narrow-strip analysis.) 
At $a=\pi$, the flat mode and the first wavy mode both lose stability at $\nu_{c}=1 / 3$, while the other modes remain stable. The nonlinear interaction of the flat and curvy modes is the subject of the weakly nonlinear analysis in [12], which culminates in the derivation of a system of two complex Landau-Stuart equations. (Notice that if the width $a$ is infinite, a continuum of modes goes unstable, and the evolution is governed by Ginzburg-Landau PDEs.)

In the remainder of this section, we introduce the governing equations and, for convenience, summarize a linear stability analysis. Because we consider the case in which a zero-wavenumber mode is the most unstable, we present the model in one space dimension. In [21], we do a full linear stability study for the two-dimensional problem formulated as an initial-value problem.

Section 2 contains a weakly nonlinear analysis, and section 3 shows simulations in the marginally unstable regime. In computations, the dynamics unfold as the parameter $\sigma$ associated with the Arrhenius kinetics decreases (while the bifurcation parameter $\nu$ remains fixed within $\epsilon^{2}$ of its neutrally stable value).

Section 4 presents quantitative comparisons of the asymptotic solutions and computed solutions. Some qualitative comparisons for a similar problem-involving competing flat and wavy (two-dimensional) modes - appear in [6] (together with numerics that venture into more strongly unstable regimes than in the present paper). Here we investigate the numerical solutions for marginally unstable values of the activation energy, allowing a full range of kinetics-parameter values.

Specifically, we perform the computations with $\nu$ fixed near the marginally unstable value, namely, $\nu=\nu_{c}-\epsilon^{2}$, where $\epsilon$ is fairly small. For $\epsilon$ smaller than about 0.12 , we see the smooth periodic solutions that the weakly nonlinear analysis predicts, provided $\sigma$ has an appropriate value. In particular, Fourier transforms of the numerical data illustrate the ranges of $\sigma$ in which the analysis accurately predicts the quantitative behavior of solutions.

The data simultaneously reveal the development of complex dynamics in various kinetics-parameter regimes (with the inverse activation energy $\nu$ held at $\epsilon^{2}$ units below the stability threshold), when $\epsilon$ exceeds about 0.06 . When $\epsilon$ drops below this value, the $\sigma$ intervals of strongly nonlinear dynamics disappear.

In the model, we seek the temperature distribution $u(x, t)$ in one spatial dimension and the interface position $\Gamma(t)=\{x \mid x=f(t)\}$ that satisfy the appropriately nondimensionalized free-boundary problem

$$
\begin{gathered}
\frac{\partial u}{\partial t}=\frac{\partial^{2} u}{\partial x^{2}}, \quad x>f(t), \quad t>0, \\
V=G\left(\left.u\right|_{\Gamma}\right), \quad t>0, \\
\left.\frac{\partial u}{\partial x}\right|_{\Gamma}=-V, \quad t>0 .
\end{gathered}
$$

Here $V$ is the velocity of the rightward-traveling interface, i.e.,

$$
V=\frac{d f}{d t} .
$$

In addition, the temperature satisfies the condition

$$
u \rightarrow 0 \text { as } x \rightarrow \infty ;
$$


that is, the ambient temperature is normalized to zero at infinity.

To model solid combustion, we take the Arrhenius function as the kinetics function $G$ in the nonequilibrium interface condition $(1.2)[4,17]$. Then, with appropriate nondimensionalization, the velocity of propagation relates to the interface temperature as

$$
V=\exp \left[\left(\frac{1}{\nu}\right) \frac{u-1}{\sigma+(1-\sigma) u}\right]
$$

at the interface $\Gamma$. Here $\nu$ is inversely proportional to the activation energy of the exothermic chemical reaction that occurs at the interface, and $0<\sigma<1$ is the ambient temperature nondimensionalized by the adiabatic temperature of combustion products. (See [8].)

Inverting the Arrhenius function (1.5), we reexpress the boundary condition (1.2) in the form

$$
\left.u\right|_{\Gamma}=1+\nu K(V ; \sigma, \nu),
$$

where

$$
K(V ; \sigma, \nu)=\frac{\ln (V)}{1-(1-\sigma) \nu \ln (V)} .
$$

Note the function $K(V)$ has been introduced to have the convenient properties $K(1)=$ $0, K^{\prime}(1)=1$.

For ease of subsequent asymptotic and numerical analysis, we reformulate the problem in the front-attached coordinate frame:

$$
\eta=x-f(t), \quad \tau=t .
$$

Problem (1.1)-(1.6) then takes the form

$$
\begin{gathered}
\frac{\partial u}{\partial \tau}=\frac{\partial^{2} u}{\partial^{2} \eta}+V \frac{\partial u}{\partial \eta}, \quad \eta>0, \tau>0, \\
\left.u\right|_{\Gamma}=u(0, \tau)=1+\nu K(V), \\
\left.\frac{\partial u}{\partial \eta}\right|_{\Gamma}=\left.\frac{\partial u}{\partial \eta}\right|_{(0, \tau)}=-V, \\
\lim _{\eta \rightarrow \infty} u=0 .
\end{gathered}
$$

The free-boundary problem (1.8)-(1.11) admits a traveling-wave solution

$$
u_{0}(\eta, \tau)=\exp (-\eta), \quad f_{0}(\tau)=\tau .
$$

The problem linearized about the traveling wave has a normal-mode solution of the form

$$
w=e^{\lambda \tau} g(\eta ; \lambda), \quad \phi=e^{\lambda \tau},
$$


where $w$ and $\phi$ represent the perturbations about $u_{0}$ and $f_{0}$, respectively. Substituting them into the linearized problem produces an eigenvalue problem in $\lambda$ and $g(\eta ; \lambda)$.

The discrete spectrum values are zero and

$$
\lambda=\frac{1-3 \nu \pm \sqrt{(3 \nu-1)^{2}-4 \nu^{3}}}{2 \nu^{2}} .
$$

The eigenfunction corresponding to the eigenvalue $\lambda$ is

$$
g(\eta ; \lambda, \nu)=(1+\nu \lambda) \exp \left(-(1+\sqrt{1+4 \lambda}) \frac{\eta}{2}\right)-\exp (-\eta) .
$$

Linearly unstable behavior occurs for this system only when $\Re \lambda$ is positive.

The basic solution (1.12) is neutrally stable under a small perturbation of the form (1.13) if $\Re \lambda=0$. Setting $\Re \lambda=0$ in (1.14) gives the critical value $\nu_{c}$ of $\nu$, namely,

$$
\nu_{c}=\frac{1}{3}
$$

The corresponding neutrally stable eigenvalues from (1.14) are $\pm i \omega$, where

$$
\omega=\sqrt{3} .
$$

If $\nu<1 / 3$, then $\Re \lambda>0$, and the basic solution is linearly unstable. (See, for example, $[12,21]$.)

2. Weakly nonlinear analysis. Let $\epsilon^{2}$ be a small deviation from the neutrally stable value of $\nu$, namely,

$$
\epsilon^{2}=\nu_{c}-\nu=\frac{1}{3}-\nu
$$

We consider the time scales

$$
t_{0}=\tau, \quad t_{1}=\epsilon \tau, \quad t_{2}=\epsilon^{2} \tau
$$

as independent variables, so that $\partial / \partial \tau=\partial / \partial t_{0}+\epsilon \partial / \partial t_{1}+\epsilon^{2} \partial / \partial t_{2}$. We then seek a solution of the form

$$
\begin{aligned}
u\left(\eta, t_{0}, t_{1}, t_{2}\right) & =e^{-\eta}+\epsilon A\left(t_{1}, t_{2}\right) e^{i \sqrt{3} t_{0}} g\left(\eta ; i \sqrt{3}, \frac{1}{3}\right) \\
& +\epsilon^{2} w_{2}\left(\eta, t_{0}, t_{1}, t_{2}\right)+\cdots+\mathrm{CC}, \\
f\left(t_{0}, t_{1}, t_{2}\right) & =t_{0}+\epsilon\left\{A\left(t_{1}, t_{2}\right) e^{i \sqrt{3} t_{0}}+\frac{1}{2} B\left(t_{1}, t_{2}\right)\right\} \\
& +\epsilon^{2} \phi_{2}\left(t_{0}, t_{1}, t_{2}\right)+\cdots+\mathrm{CC},
\end{aligned}
$$

where $A\left(t_{1}, t_{2}\right)$ is complex, and "CC" stands for complex-conjugate terms. The realvalued function $B\left(t_{1}, t_{2}\right)$ modulates the constant-velocity solution to the linearized problem.

Notice that in $O(\epsilon)$, the weakly nonlinear solution (2.2) has only one Fourier term in $t_{0}$. We will show below in $(2.19)-(2.20)$ that the $O\left(\epsilon^{2}\right)$ term contains the second harmonic. We refer to the expansion (2.2) as a "single-mode approximation" because the leading-order perturbation contains only one mode in fast time. 
Making the substitutions (2.2) and equating like powers of $\epsilon$ results in subproblems for the terms in the perturbation expansions above, subject to solvability conditions on the amplitudes $A$ and $B$. The $O(1)$ problem is satisfied identically because in (2.2) we took the temperature-interface pair $(u, f)$ perturbed about $\left(e^{-\eta}, t_{0}\right)$, a solution to the nonlinear problem $(1.8)-(1.11)$. The $O(\epsilon)$ problem is just the linearized problem with $\nu=\nu_{c}=1 / 3$, which is satisfied identically by the $O(\epsilon)$ terms in the expansions (2.2).

The problems of order $\epsilon^{j}, j=2,3$, are

$$
\begin{aligned}
\frac{\partial w_{j}}{\partial t_{0}}-\frac{\partial^{2} w_{j}}{\partial \eta^{2}}-\frac{\partial w_{j}}{\partial \eta}+e^{-\eta} \frac{\partial \phi_{j}}{\partial t_{0}} & =Q_{j}(\eta, \mathbf{t}), \\
\left.w_{j}\right|_{\eta=0}-\frac{1}{3} \frac{\partial \phi_{j}}{\partial t_{0}} & =\alpha_{j}(\mathbf{t}), \\
\left.\frac{\partial w_{j}}{\partial \eta}\right|_{\eta=0}+\frac{\partial \phi_{j}}{\partial t_{0}} & =\beta_{j}(\mathbf{t}), \\
\lim _{\eta \rightarrow \infty} w_{j} & =0
\end{aligned}
$$

where $\mathbf{t}=\left(t_{0}, t_{1}, t_{2}\right)$. For brevity, we have named the right-hand sides above as $Q_{j}$, $\alpha_{j}$, and $\beta_{j}$. The PDEs (2.3) can be represented as

$$
\mathcal{L}_{1} w_{j}+\mathcal{L}_{2} \phi_{j}=\mathcal{P}\left(w_{1}, \phi_{1}, \ldots, w_{j-1}, \phi_{j-1}\right) .
$$

$\mathcal{L}_{1}$ and $\mathcal{L}_{2}$ are linear operators on bounded functions in $L^{2}(\Omega)$, where $\Omega=\{(\eta, \tau) \mid 0 \leq$ $\eta<\infty, 0 \leq \tau<\infty\}$.

According to Fredholm's alternative, equation (2.7) has a nonsecular (boundedin-time) solution if the right-hand side is orthogonal to the null space of the adjoint operator $\mathcal{L}^{*}$. That is,

$$
\left(\mathcal{L}_{1} w_{j}+\mathcal{L}_{2} \phi_{j}, v\right)=0
$$

for $v \in \operatorname{ker} \mathcal{L}^{*}$ and the inner product defined such that

$$
\left(f_{1}, f_{2}\right)=\lim _{T \rightarrow \infty} \frac{1}{T} \int_{0}^{T} \int_{0}^{\infty} f_{1}(\eta, \tau) \overline{f_{2}(\eta, \tau)} d \eta d \tau .
$$

The quantity $v$ in (2.8) satisfies

$$
\begin{gathered}
\left(-\frac{\partial}{\partial t_{0}}-\frac{\partial^{2}}{\partial \eta^{2}}+\frac{\partial}{\partial \eta}\right) v=0, \\
\left.2 i \frac{\sqrt{3}}{3} v\right|_{\eta=0}-\left.\left(1-i \frac{1}{3}\right) \frac{\partial v}{\partial \eta}\right|_{\eta=0}=0 .
\end{gathered}
$$

Nonzero solutions are

$$
u_{1}\left(\eta, t_{0} ; i \sqrt{3}\right)=\exp \left(i \sqrt{3} t_{0}\right) h(\eta ; i \sqrt{3}) \text { and } u_{0}\left(\eta, t_{0} ; 0\right)=1,
$$

where

$$
h(\eta ; i \sqrt{3})=\exp \left((1-\sqrt{1-4 i \sqrt{3}}) \frac{\eta}{2}\right)
$$


Substituting $v=u_{1}$ into the solvability condition (2.8) when $j=2$ produces the differential equation

$$
\frac{\partial A}{\partial t_{1}}=0 .
$$

Substituting $v=u_{0}$ into the solvability condition (2.8) when $j=2$ produces the differential equation

$$
\frac{\partial B}{\partial t_{1}}=A \bar{A} r_{0}, \quad r_{0}=-3\left(2+K^{\prime \prime}(1)\right) .
$$

Substituting (2.14) and (2.15) into the $O\left(\epsilon^{2}\right)$ problem in (2.3)-(2.6) yields the problem

$$
\begin{aligned}
\frac{\partial w_{2}}{\partial t_{0}}-\frac{\partial^{2} w_{2}}{\partial \eta^{2}}-\frac{\partial w_{2}}{\partial \eta}+e^{-\eta} \frac{\partial \phi_{2}}{\partial t_{0}} & =A^{2} e^{2 i \sqrt{3} t_{0}} \mathcal{R}_{2}(\eta)+A \bar{A} \mathcal{R}_{0}(\eta)+\mathrm{CC} \\
\left.w_{2}\right|_{\eta=0}-\frac{1}{3} \frac{\partial \phi_{2}}{\partial t_{0}} & =A^{2} e^{2 i \sqrt{3} t_{0}} F_{2}+A \bar{A} F_{0}+\mathrm{CC} \\
\left.\frac{\partial w_{2}}{\partial \eta}\right|_{\eta=0}+\frac{\partial \phi_{2}}{\partial t_{0}} & =A^{2} e^{2 i \sqrt{3} t_{0}} G_{2}+A \bar{A} G_{0}+\mathrm{CC}
\end{aligned}
$$

The solution $\left(w_{2}, \phi_{2}\right)$ consists of a homogeneous and a particular solution. Because only the inhomogeneous terms will contribute to the solvability condition at the next order, we present the nonsecular solution as

$$
\begin{aligned}
& w_{2}=A^{2} e^{2 i \sqrt{3} t_{0}} g_{2}(\eta)+A \bar{A} g_{0}(\eta)+\mathrm{CC}, \\
& \phi_{2}=A^{2} e^{2 i \sqrt{3} t_{0}} C_{2}+A \bar{A}+\mathrm{CC},
\end{aligned}
$$

where $g_{j}(\eta), j=0,2$, satisfy the initial-value problems

$$
\begin{gathered}
g_{j}^{\prime \prime}+g_{j}^{\prime}-j i \sqrt{3} g_{j}=j i \sqrt{3} C_{2} e^{-\eta}-\mathcal{R}_{j}(\eta), \\
g_{j}(0)=j i \frac{\sqrt{3}}{3} C_{2}+F_{j}, \\
g_{j}^{\prime}(0)=-j i \sqrt{3} C_{2}+G_{j}, \\
g_{2}(\eta) \rightarrow 0 \text { as } \eta \rightarrow \infty
\end{gathered}
$$

where

$$
\begin{gathered}
\mathcal{R}_{j}(\eta)=-(-1)^{j / 2} g^{\prime}(\eta) i \sqrt{3}-\frac{2-j}{4} r_{0} e^{-\eta} \\
F_{j}=\frac{1}{2}(-1)^{j / 2} K^{\prime \prime}(1)+\frac{2-j}{12} r_{0} \\
G_{j}=-\frac{2-j}{4} r_{0} .
\end{gathered}
$$


Recall that $r_{0}$ is given in (2.15). Also, $g(\eta)=g(\eta ; i \sqrt{3})$ is defined via (1.15).

Substituting $v=u_{1}$ into the solvability condition (2.8) when $j=3$ produces the Landau-Stuart equation

$$
\frac{d A}{d t_{2}}=\chi A+\beta A^{2} \bar{A}
$$

where

$$
\chi \equiv-\left.\frac{\partial \lambda_{0}}{\partial \nu}\right|_{\nu=\nu_{c}}=\frac{3}{2}(9+\sqrt{3} i) .
$$

The coefficient $\beta$ is defined as

$$
\beta=\frac{\int_{0}^{\infty} R(\eta) \bar{h}(\eta) d \eta+F \mathcal{U}}{\int_{0}^{\infty}\left(g(\eta)+e^{-\eta}\right) \bar{h}(\eta) d \eta-\frac{1}{3} \mathcal{U}-1},
$$

where

$$
\begin{aligned}
R(\eta) & =r_{0} g^{\prime}(\eta)+i \sqrt{3}\left[2 C_{2} \bar{g}^{\prime}(\eta)-g_{2}^{\prime}(\eta)+2 \operatorname{Re}\left(g_{0}^{\prime}(\eta)\right)\right] ; \\
F & =\frac{1}{3}\left(6 C_{2}+i r_{0} \sqrt{3}\right) K^{\prime \prime}(1)+i \frac{\sqrt{3}}{2} K^{\prime \prime \prime}(1) ; \\
\mathcal{U} & =-\frac{1}{2}(3+i \sqrt{3}) .
\end{aligned}
$$

Once we solve the Landau-Stuart equation (2.28) subject to an initial condition, the full asymptotic expansion (2.2) is known with $w_{2}$ and $\phi_{2}$ given in (2.19)-(2.20) and $B$ given in (2.15). In what follows, we compare the asymptotic solution with a numerical solution over the range $0<\sigma<1$ with $\nu$ fixed at a small deviation $\epsilon^{2}$ from the neutrally stable value $1 / 3$, as given in (2.1).

The amplitude equation (2.28) determines the dynamics of the unstable mode $A\left(t_{2}\right) e^{i \sqrt{3} t_{0}}$, subject to self-interaction. The dynamics of the mode depend on the relationships between the coefficients $\chi$ and $\beta$ and are affected by the kinetics function $K(V)$, introduced in (1.7). Recall that the function $K(V)$ is normalized such that $K(1)=0$ and $K^{\prime}(1)=1$. The form of the kinetics function comes into play via $K^{\prime \prime}(1)$ and $K^{\prime \prime \prime}(1)$, which appear explicitly in $r_{0}, F_{j}$, and $F$ of (2.15), (2.26), and (2.32), respectively. Note from (2.17) that $F_{j}, j=2,0$, are the coefficients of $A^{2} e^{2 i \sqrt{3} t_{0}}$ and $A \bar{A}$, respectively, on the right-hand side of an $O\left(\epsilon^{2}\right)$ boundary condition. Also, $F$ is the coefficient of $A^{2} \bar{A} e^{i \sqrt{3} t_{0}}$ on the right-hand side $\alpha_{3}(\mathbf{t})$ of the $O\left(\epsilon^{3}\right)$ boundary condition (2.4). In particular, $\alpha_{3}(\mathbf{t})$ has the form

$$
\begin{aligned}
\alpha_{3}(\mathbf{t})= & \left\{\left(\frac{\partial A}{\partial t_{2}}-\chi A\right) \frac{1}{3} e^{i \sqrt{3} t_{0}}\right. \\
& \left.+A^{3} e^{3 i \sqrt{3} t_{0}} F_{3}+A^{2} \bar{A} e^{i \sqrt{3} t_{0}} F+\mathrm{CC}\right\}+\frac{1}{3} \frac{\partial B}{\partial t_{2}} .
\end{aligned}
$$

( $F_{3}$ does not pertain to this discussion.)

To examine the behavior of the front in the different parameter regimes, let us consider the real equation in $|A|$ corresponding to the complex equation (2.28), namely,

$$
\frac{d|A|}{d t_{2}}=|A|\left(\operatorname{Re}(\chi)+|A|^{2} \operatorname{Re}(\beta)\right) .
$$


Perturbation amplitude $|A|=0$ is a stationary solution for (2.35). Because $\operatorname{Re}(\chi)=27 / 2$ is greater than zero, trajectories with an initial point near the origin in the complex $A$ plane tend away from the origin. That is, in the absence of other equilibria, the amplitude blows up in (slow) time for $\nu$ slightly below the critical value $1 / 3$.

Equation (2.35) has a second equilibrium $|A|=\sqrt{-\operatorname{Re}(\chi) / \operatorname{Re}(\beta)}$ (a circle in the complex- $A$ plane) if $\operatorname{Re}(\beta)$ is negative. A simple stability analysis of (2.35) shows that $d|A| / d t_{2}<0$ outside of the circle, and $d|A| / d t_{2}>0$ inside it. As a result, the limit cycle in the complex- $A$ plane is asymptotically stable in this setting. A supercritical Hopf bifurcation occurs at $\nu=1 / 3$. The nonlinear solution develops oscillations of magnitude $O(\epsilon)$ on the time scale $O\left(\epsilon^{-2}\right)$. (See the expansion in (2.2).)

The quantity $\operatorname{Re}(\beta)$ is a quadratic function in $\sigma$ with no roots at physical values of $\sigma$. For all $0<\sigma<1, \operatorname{Re}(\beta)$ is negative. The amplitude of the flat mode $A\left(t_{2}\right) e^{i \sqrt{3} t_{0}}$ approaches the limit cycle $|A|=\sqrt{-\operatorname{Re}(\chi) / \operatorname{Re}(\beta)}$. The nonlinear problem (1.8)(1.11) develops oscillations, as detailed below.

3. Numerical method. We integrate numerically the exact problem as given by (1.8)-(1.11). In section 4 , we compare the numerical solution with the asymptotics derived above. As was pointed out in [7], numerical solutions of (1.8)-(1.11) are very sensitive to the boundary condition (1.10). In order to obtain an alternative condition, we integrate (1.8) with respect to $\eta$ from 0 to $\infty$. Subsequently applying conditions (1.9)-(1.11) results in the equation

$$
\frac{d}{d t} \int_{0}^{\infty} u d \eta=-\nu f_{t} K(V)
$$

We use (3.1) to replace (1.10) and adopt the Crank-Nicolson method for the numerical solution. The computation domain for $\eta$ is $[0,10]$ with $\delta t=\delta \eta=0.025$. This produces a nonlinear system of $m(=401)$ equations. In particular, in reference to $(2.2)$, we introduce perturbation variables $u^{*}$ and $f^{*}$ defined by

$$
u=e^{-\eta}+\epsilon u^{*} ; \quad f=t+\epsilon f^{*} .
$$

Our discretization of condition (3.1) is

$$
\begin{aligned}
\left.\left(\int_{0}^{\infty} u^{*} d \eta\right)\right|_{t_{k}} ^{t_{k+1}}=-\frac{\nu}{2 \epsilon} & {\left[\left(1+\epsilon f_{t}^{*}\left(t_{k+1}\right)\right) K\left(1+\epsilon f_{t}^{*}\left(t_{k+1}\right)\right)\right.} \\
& \left.+\left(1+\epsilon f_{t}^{*}\left(t_{k}\right)\right) K\left(1+\epsilon f_{t}^{*}\left(t_{k}\right)\right)\right]\left(t_{k+1}-t_{k}\right)
\end{aligned}
$$

where the integral on the left-hand side of (3.3) can be approximated by a composite trapezoidal rule.

We solve the nonlinear system of equations using Newton's method. The Jacobian matrix has the following sparse structure:

$$
\left(\begin{array}{cccccccc}
\# & \# & 0 & 0 & 0 & 0 & \ldots & 0 \\
\# & \# & \# & \# & \# & \# & \ldots & \# \\
\# & \# & \# & \# & 0 & 0 & \ldots & 0 \\
\# & 0 & \# & \# & \# & 0 & \ldots & 0 \\
\vdots & & & \ddots & \ddots & \ddots & & \vdots \\
\# & 0 & \ldots & 0 & \# & \# & \# & 0 \\
\# & 0 & \ldots & 0 & 0 & \# & \# & \# \\
\# & 0 & \ldots & 0 & 0 & 0 & \# & \#
\end{array}\right),
$$


where \# denotes a nonzero element. The matrix can be efficiently inverted by using Gaussian elimination with backward substitution.

The next section contains comparisons between numerical and asymptotic solutions. For the asymptotic solution, we integrate the ordinary differential equation (2.28) using a fourth-order Runge-Kutta method. As was pointed out in section 2, the Landau-Stuart equation (2.28) has circular limit cycles in the complex- $A$ plane for all values of the kinetic parameter $\sigma$ in the interval $0<\sigma<1$.

4. Comparison between asymptotics and numerics. To fix the idea, we first consider $\epsilon=0.1$. The value of $\nu$ remains at the marginally unstable value $\nu_{c}-\epsilon^{2}$, as introduced in (2.1), so $\nu \approx 0.32 \overline{3}$. We show in this section that this choice of $\epsilon$ corresponds to a mix of dynamics as $\sigma$ varies. Subsequently, we both decrease and increase $\epsilon$ and discuss the impact on the front behavior. For the remainder of this paper, we take the initial condition $A(0)=0.1$.

To start, take $\sigma=0.48$ in the kinetic function (1.7). Figure 4.1 shows the numerical (solid line) and asymptotic (dashed line) values of front speed perturbation as a function of time $t$ in the interval $0 \leq t \leq 60$. Specifically, for the numerical and asymptotic solutions we have graphed the quantities

$$
v_{n}=f_{t}^{*} \text { and } v_{a}=A\left(t_{2}\right) e^{i \sqrt{3} t_{0}}+\frac{1}{2} B\left(t_{1}, t_{2}\right)+\epsilon \phi_{2}\left(t_{0}, t_{1}, t_{2}\right),
$$

respectively, where $f^{*}$ is defined in (3.2), and $v_{a}$ contains the first three terms in the perturbation in (2.2).

Figure 4.1 shows that from $t=0$ to about $t=30$, the small front speed perturbation is linearly unstable, and its amplitude grows exponentially in time. As this amplitude becomes large, nonlinearity takes effect. At around $t=30$, the front speed

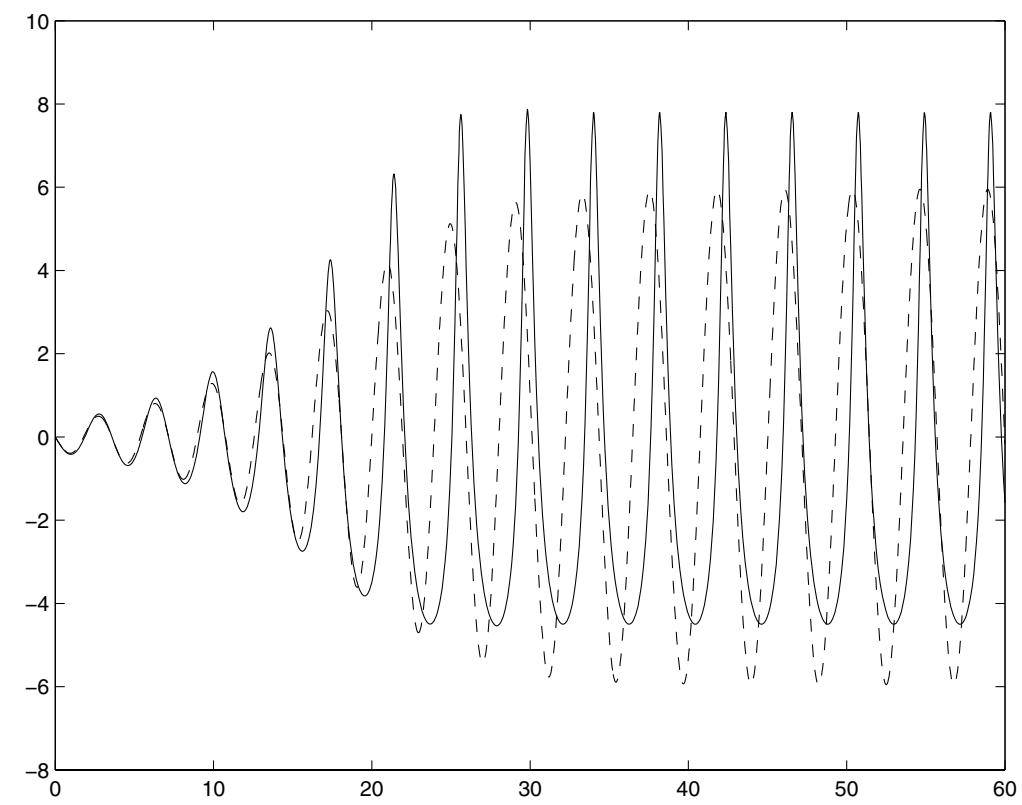

FIG. 4.1. Velocity perturbation versus time: Comparison between numerical (solid line) and asymptotic (dashed line); $\sigma=0.48, \epsilon=0.1, A(0)=0.1\left(\nu \approx \nu_{c}-\epsilon^{2}=1 / 3-(0.1)^{2}=0.32 \overline{3}\right)$. 


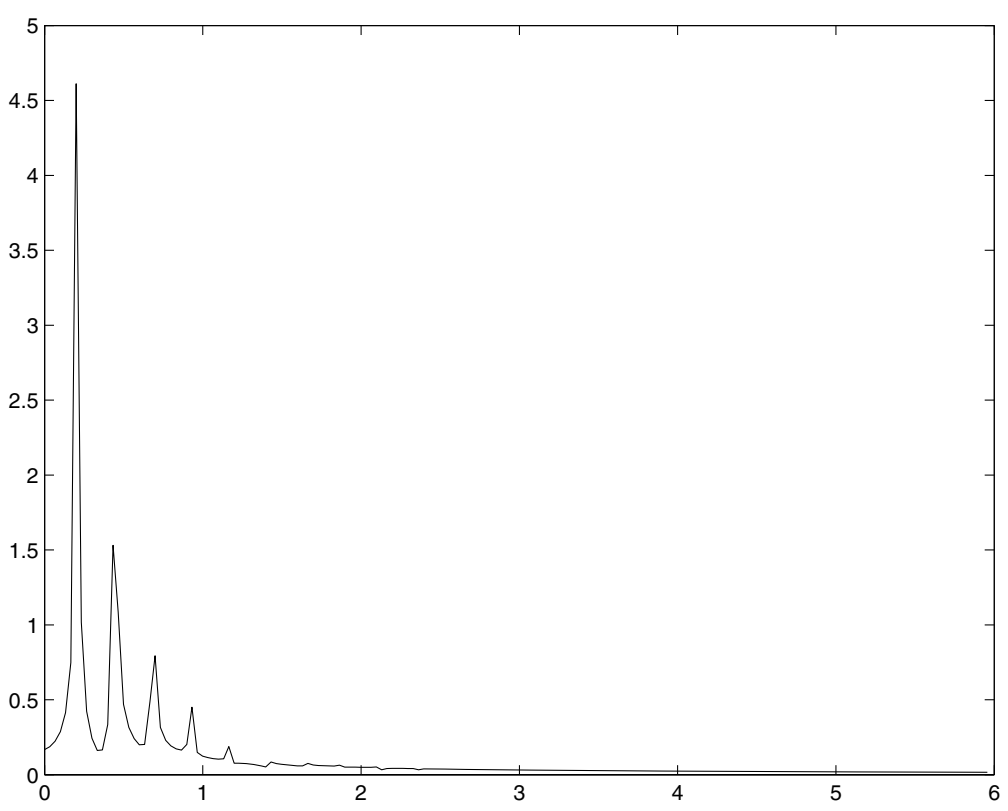

FIG. 4.2. Fourier amplitude of the numerical steady-state velocity perturbation; $\sigma=0.48$, $\epsilon=0.1, A(0)=0.1,50<t<100\left(\nu \approx \nu_{c}-\epsilon^{2}=1 / 3-(0.1)^{2}=0.32 \overline{3}\right)$.

perturbation has reached steady oscillation. The asymptotic solution accurately captures the period in both transient behavior for $t=0$ to 30 and the long-time behavior after $t=30$. The amplitude and phase differ somewhat.

The weakly nonlinear approach describes well, by definition, marginally unstable large-time behaviors when a single modulated temporal mode of frequency $\sqrt{3}$ captures the dynamics. (See (2.2).) We have illustrated such a case in Figure 4.1. We then numerically calculated the velocity perturbation data $\left\{f_{t}^{*}\left(t_{i}\right)\right\}$ on the time interval $50<t<100$, using the parameter values as in Figure 4.1. The discrete Fourier transform of the data reveals the dominance of one mode. (See Figure 4.2.)

However, the subsequent modes do contribute to the solution as well. The second spike in Figure 4.2 is about $3 / 5$ the height of the first, and the third is fully $1 / 2$ the height of the second. Contributions of higher-order modes may explain some quantitative discrepancies between the numerical and asymptotic solutions in Figure 4.1.

Figure 4.3 summarizes the Fourier transformed velocity data for all physical values of $\sigma(0<\sigma<1)$. For each $\sigma$ value and each frequency, the color indicates the corresponding amplitude, with the red end of the spectrum standing for larger numbers than the violet end, as the legend to the right of the figure illustrates. For roughly $0.3<\sigma<0.6$, the figure shows the dominance of the lowest-order mode, suggesting the appropriateness of the weakly nonlinear analysis in this range.

Notice, however, that at least four additional modes appear significant as well. Nevertheless, for $\sigma$ in the interval approximately $(0.3,0.6)$, the weakly nonlinear solution captures the gross features of the oscillation. (See, for example, Figure 4.1.)

With our choice of $\epsilon=0.1$, Figure 4.3 shows that for $\sigma$ greater than approximately 0.6 , a single mode cannot be expected to capture the full dynamics of the solution. For example, for $\sigma=0.85$, the asymptotic solution certainly will not exhibit a velocity perturbation with the very sharp peaks seen in the numerical solution in Figure 4.4. 


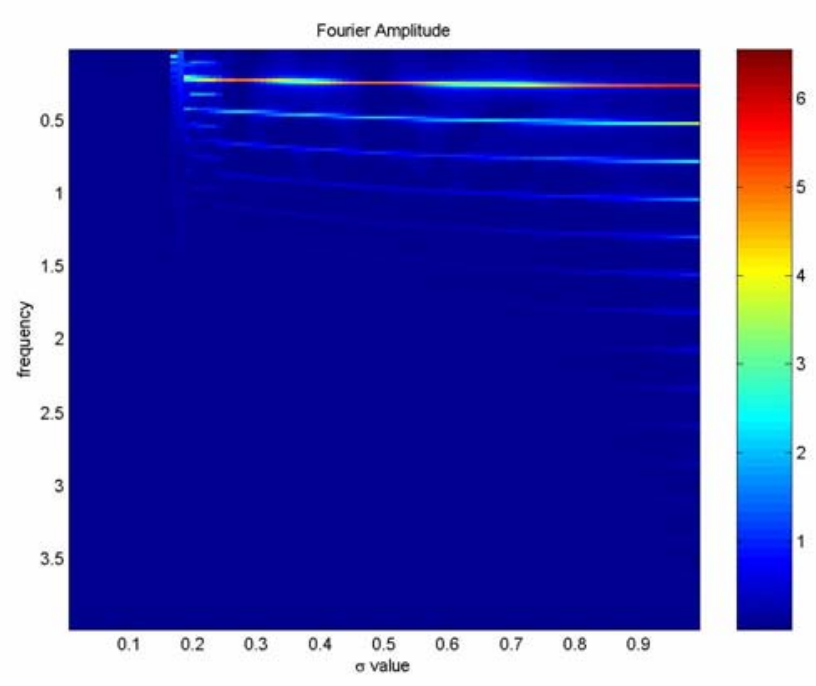

FIG. 4.3. Amplitudes corresponding to each frequency of the Fourier transformed velocity perturbation data for the Arrhenius kinetics parameter $\sigma$ in the interval $(0,1) ; \epsilon=0.1, A(0)=0.1$, $35<t<85\left(\nu \approx \nu_{c}-\epsilon^{2}=1 / 3-(0.1)^{2}=0.32 \overline{3}\right)$.

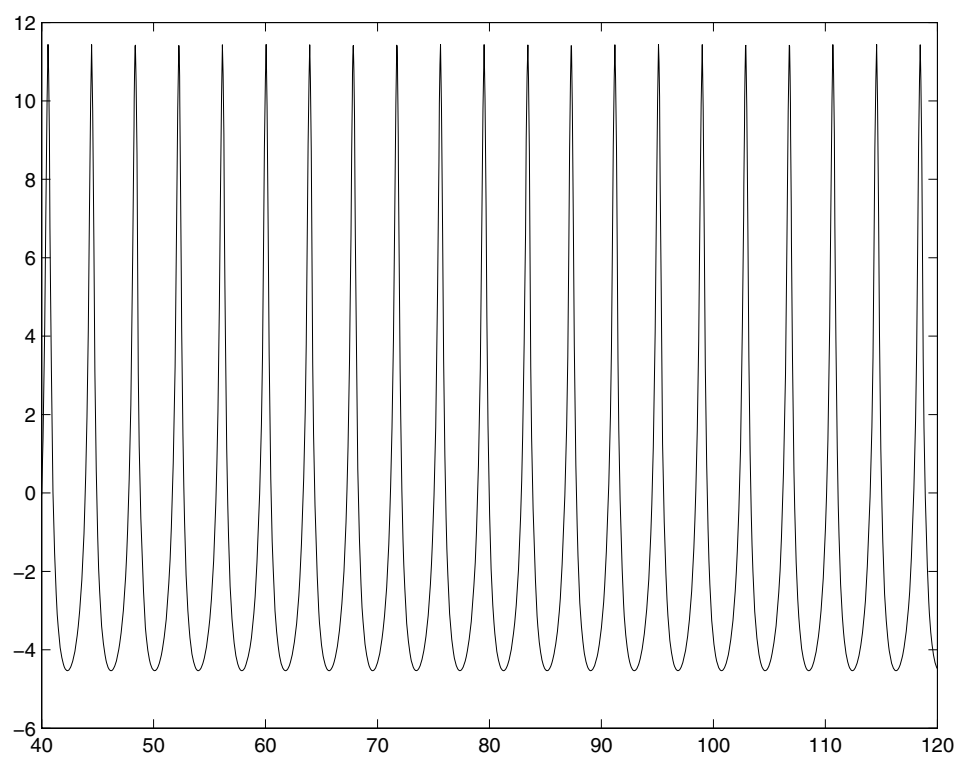

FIG. 4.4. Velocity perturbation versus time: Numerical solution for $\sigma=0.85, \epsilon=0.1, A(0)=$ $0.1\left(\nu \approx \nu_{c}-\epsilon^{2}=1 / 3-(0.1)^{2}=0.32 \overline{3}\right)$.

Further, Figure 4.3 shows that the Fourier spectrum has a complicated character for $\sigma$ sufficiently small, starting with the emergence of a period-doubling solution for $\sigma \approx 0.25$. Naturally, the asymptotic solution captures neither the period-doubling solution nor the period-quadrupling computed for $\sigma=0.22$ and $\sigma=0.21$, respectively. (Numerical solutions in Figures 4.5 and 4.6 illustrate the dynamics.) Figure 4.3 reflects the breakdown of the numerical solution for $\sigma$ less than approximately 0.15 . 


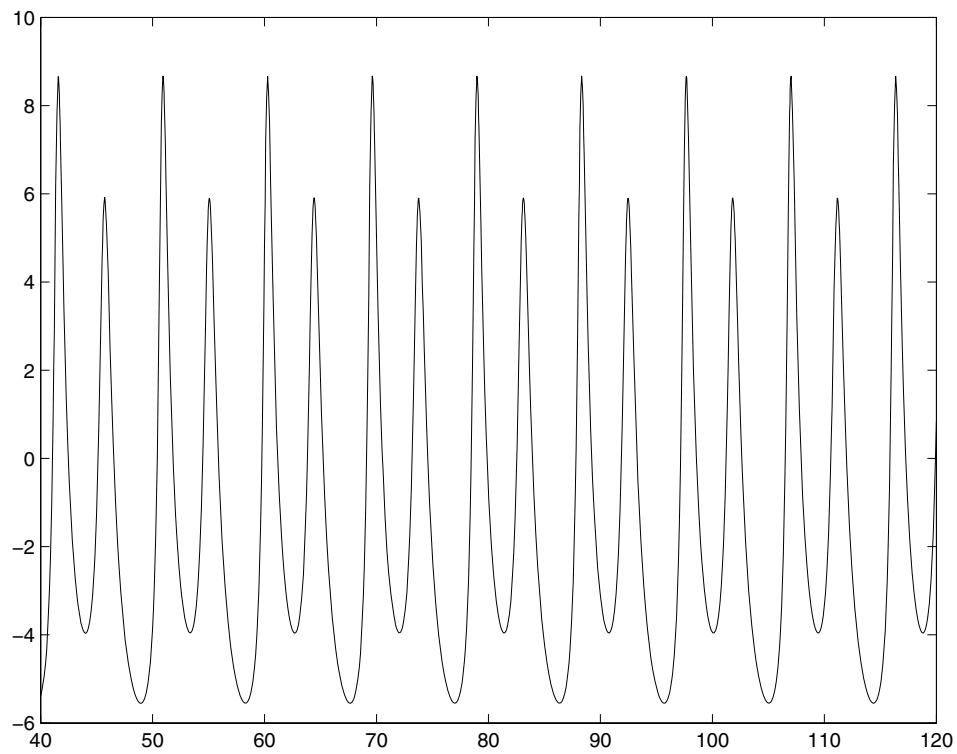

FIG. 4.5. Velocity perturbation versus time: Period-doubling numerical solution for $\sigma=0.22$, $\epsilon=0.1, A(0)=0.1\left(\nu \approx \nu_{c}-\epsilon^{2}=1 / 3-(0.1)^{2}=0.32 \overline{3}\right)$.

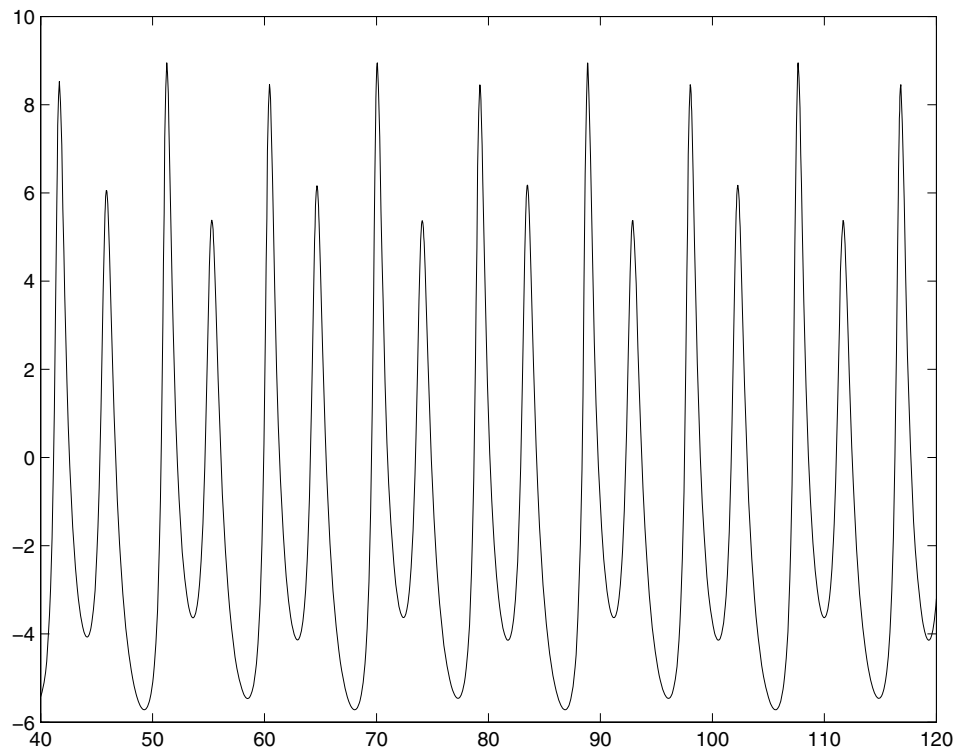

FIG. 4.6. Velocity perturbation versus time: Period-quadrupling numerical solution for $\sigma=$ $0.21, \epsilon=0.1, A(0)=0.1\left(\nu \approx \nu_{c}-\epsilon^{2}=1 / 3-(0.1)^{2}=0.32 \overline{3}\right)$.

The weakly nonlinear analysis of section 2 predicts periodic single-mode-dominant solutions for all physical values of $\sigma(0<\sigma<1)$ when $\nu=1 / 3-\epsilon^{2}$ is sufficiently close to the neutrally stable value $\nu_{c}=1 / 3$. From numerical simulation with $\epsilon=0.1$, the interval in which a single mode dominates has been identified via Figure 4.3 as a subinterval of $(0,1)$, namely, $(0.3,0.6)$. The corresponding asymptotic solution 


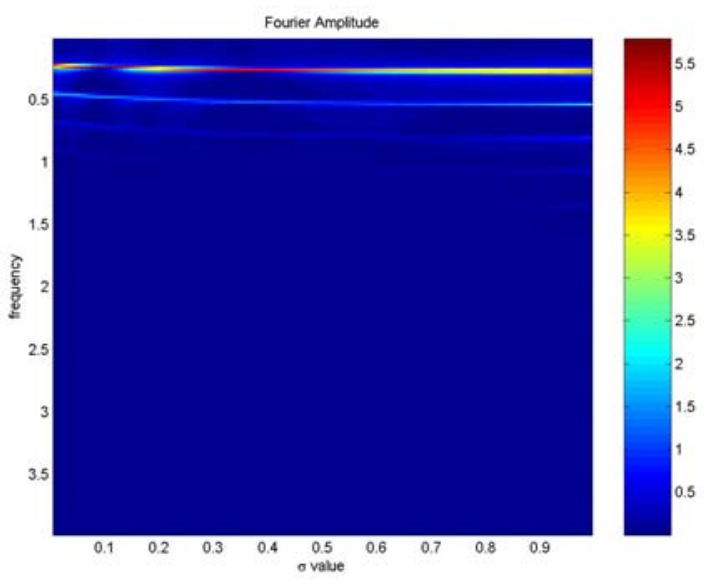

FIG. 4.7. Amplitudes corresponding to each frequency of the Fourier transformed velocity perturbation data for the Arrhenius kinetics parameter $\sigma$ in the interval $(0,1) ; \epsilon=0.06, A(0)=0.1$, $35<t<85\left(\nu \approx \nu_{c}-\epsilon^{2}=1 / 3-(0.06)^{2}\right)$.

captures the numerical solution accurately even for $\epsilon$ as large as 0.1 when the dynamics associated with varying the parameter $\sigma$ are not too complex. In particular, the interval $0.3<\sigma<0.6$ corresponds to good agreement. In what follows, we discuss the effects of decreasing and increasing $\epsilon$.

We have seen that the approximate interval of $\sigma \geq 0.6$ in Figure 4.3 corresponds to sharply spiking solutions with many Fourier modes contributing. This far right interval moves farther and farther to the right as $\epsilon$ decreases. When $\epsilon$ drops to 0.06 , the interval of spiking solutions disappears.

As we decrease $\epsilon$, graphs analogous to Figure 4.3 also show the period-doubling region pushed farther and farther to the left along the $\sigma$ axis. Similarly, the far left code-failure region moves farther to the left. When $\epsilon$ drops to 0.07 , the period-doubling interval essentially disappears, and solutions can be computed even for extremely small $\sigma$ values.

Figure 4.7 shows that for $\epsilon=0.06$, one mode dominates strongly throughout the entire interval $0<\sigma<1$. The asymptotic and numerical solutions are consistent for all physical values of $\sigma$ when $\nu=1 / 3-\epsilon^{2}$ if $\epsilon$ lies in the relatively small interval $0<\epsilon<0.06$.

Figure 4.7 also shows that only three higher-order modes appear to make slight additional contributions, fewer than for any value of $\sigma$ illustrated in Figure 4.3 for $\epsilon=0.1$. Therefore, as expected, the weakly nonlinear and numerical solutions agree more closely with $\epsilon$ reduced from 0.1 to 0.06 . Figure $4.8(\epsilon=0.06)$ shows good agreement in period - as does Figure 4.1 when $\epsilon=0.1$. Also, the phase, amplitude, and centerline agreement has improved considerably in Figure 4.8 for the decreased $\epsilon$. In Figure 4.8, the asymptotic solution oscillates between about -6 and 6 , while the numerical extends from -5 to 7 , and their difference at the quasi-steady-state peaks is about 1. In Figure 4.1 for $\epsilon=0.1$, the asymptotic solution also lies between -6 and 6 , but the numerical solution varies between -4.5 to 7.9 . The difference at the peaks is about 2 .

We have discussed the impact of reducing $\epsilon$ from the value 0.1 used in Figures 4.14.6, which all pertain to the dynamics when $\nu=1 / 3-(0.1)^{2}$. If $\epsilon$ increases beyond 0.1 , 


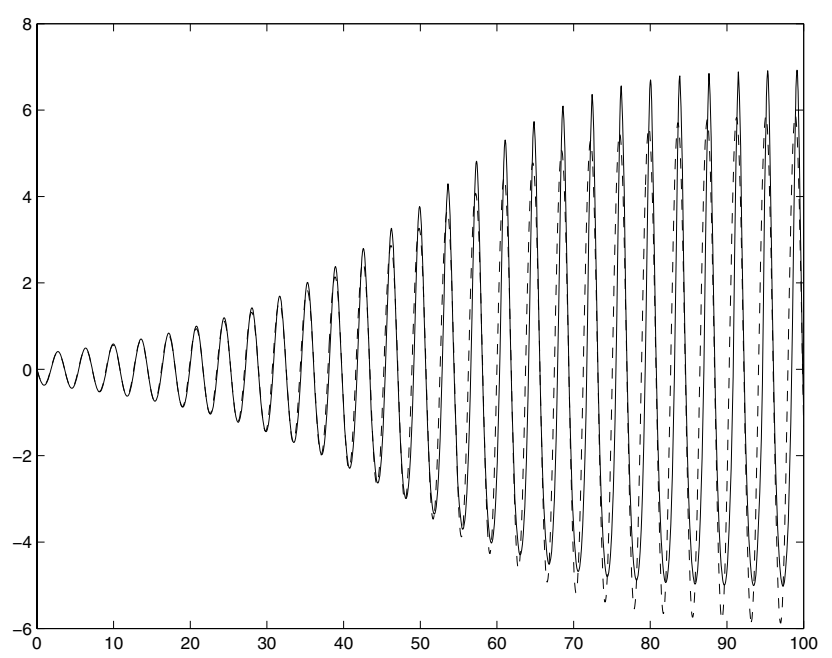

FIG. 4.8. Velocity perturbation versus time: Comparison between numerical (solid line) and asymptotic (dashed line), $\sigma=0.48, \epsilon=0.06, A(0)=0.1\left(\nu \approx \nu_{c}-\epsilon^{2}=1 / 3-(0.06)^{2}=0.3297 \overline{3}\right)$.

then the far right $\sigma$ interval in Figure 4.3, corresponding to sharply spiking solutions, has a left-hand endpoint that moves farther and farther to the left: The $\sigma$ interval of solutions with sharp peaks expands.

Also, as we increase $\epsilon$, graphs analogous to Figure 4.3 show the period-doubling region pushed farther and farther to the right along the $\sigma$ axis, when compared with Figure 4.3. In addition, the far left code-failure region has a right-hand endpoint that moves farther and farther to the right: The $\sigma$ interval on which the code breaks down expands.

When $\epsilon$ grows to 0.12 , the $\sigma$ zone in which one mode dominates strongly becomes extremely narrow. The asymptotic and numerical solutions agree well for $\sigma$ in the approximate interval $(0.41,0.42)$.

5. Conclusions and discussion. We have quantitatively compared a weakly nonlinear analysis and direct numerical integration for a solid combustion model. By definition, the weakly nonlinear approach is well suited to the study of marginally unstable large-time behaviors when the modulated most-unstable mode captures the dynamics.

For both the asymptotic and numerical methods, we examined nonuniform solutions corresponding to $\nu$ fixed within $\epsilon^{2}$ of the stability boundary. When $\epsilon=0.1$, for values of $\sigma$ in the approximate interval $(0.3,0.6)$, the weakly nonlinear analysis predicted accurately the transient and steady-state behaviors and particularly the period of oscillation. Beyond these special values of $\sigma$, the steady-state front propagation exhibited complicated nonlinear behaviors. We took the Fourier transforms of computational solutions to illustrate that higher-order modes play a significant role on $\sigma$ intervals outside of $(0.3,0.6)$ when $\epsilon=0.1$.

For larger values of $\epsilon$, the $\sigma$ interval of applicability of the weakly nonlinear analysis shrinks. By contrast, when $\epsilon$ drops to approximately 0.06 , the asymptotic and numerical solutions agree well for all physical values of $\sigma$.

Specifically, as $\epsilon$ increases from 0.06 (Figure 4.7) to $\epsilon=0.1$ (Figure 4.3) and beyond, a period-doubling sequence develops in $\sigma$. As $\epsilon$ gets larger (thereby pushing 
$\nu$ somewhat deeper into the instability region), the period-doubling bifurcation occurs at larger and larger values of $\sigma$, and the subsequent bifurcations occur as $\sigma$ decreases. We note that this result, when viewed for a fixed value of $\sigma$, shows a period-doubling sequence in $\nu$ (as $\epsilon$ increases), which concurs with the dynamical scenarios described in the literature for experiments, as well as for simulations on reaction-diffusion and free-interface models.

In future work, we will suggest a hybrid expansion-perturbation technique for capturing more complex dynamics than those that have single-mode dominance. A more flexible general expansion as in [22] will assume that the temperature and interface position can be represented as a Fourier-like series that includes multiple temporal modes varying in fast time.

Acknowledgments. The authors would like to express their gratitude to the editor Stephen B. Margolis and to the referees for very insightful comments and suggestions. We also thank Yi Yang of the Institute of Applied Physics and Computational Mathematics in Beijing for helpful recommendations.

\section{REFERENCES}

[1] A. Bayliss And B. J. Matkowsky, Two routes to chaos in condensed phase combustion, SIAM J. Appl. Math., 50 (1990), pp. 437-459.

[2] A. F. Belyaev and L. D. Komkova, Dependence of burning velocity of thermites on pressure, Zh. Fiz. Khim., 24 (1950), pp. 1302-1311.

[3] M. R. Воотy, S. B. Margolis, and B. J. Matkowsky, Interaction of pulsating and spinning waves in condensed phase combustion, SIAM J. Appl. Math., 46 (1986), pp. 801-843.

[4] I. Brailovsky and G. Sivashinsky, Chaotic dynamics in solid fuel combustion, Phys. D, 65 (1993), pp. 191-198.

[5] P. Dimitriou, J. Puszinski, and V. Hlavacek, On the dynamics of equations describing gasless combustion, Combust. Sci. Tech., 68 (1989), pp. 101-111.

[6] M. L. Frankel, L. K. Gross, and V. Roytburd, Thermo-kinetically controlled pattern selection, Interfaces Free Bound., 2 (2000), pp. 313-330.

[7] M. L. Frankel AND V. Roytburd, Dynamical portrait of a model of thermal instability: Cascades, chaos, reversed cascades, and infinite period bifurcations, Internat. J. Bifur. Chaos Appl. Sci. Engrg., 4 (1994), pp. 579-593.

[8] M. Frankel, V. Roytburd, and G. Sivashinsky, A sequence of period doublings and chaotic pulsations in a free boundary problem modeling thermal instabilities, SIAM J. Appl. Math, 54 (1994), pp. 1101-1112.

[9] M. L. Frankel, V. Roytburd, and G. Sivashinsky, Complex dynamics generated by a sharp interface model of self-propagating high-temperature synthesis, Combust. Theory Model., 2 (1998), pp. 1-18.

[10] M. Garbey, H. G. Kaper, G. K. Leaf, and B. J. Matkowsky, Quasi-periodic waves and the transfer of stability in condensed-phase surface combustion, SIAM J. Appl. Math., 52 (1992), pp. 384-395.

[11] L. K. Gross, Weakly Nonlinear Dynamics of Interface Propagation, Ph.D. thesis, Rensselaer Polytechnic Institute, Troy, NY, 1997.

[12] L. K. Gross, Weakly nonlinear dynamics of interface propagation, Stud. Appl. Math., 108 (2002), pp. 323-350.

[13] S. B. Margolis, Transition to nonsteady deflagration in gasless combustion, Progr. Energy Combust. Sci., 17 (1991), pp. 135-162.

[14] B. J. Matkowsky and G. I. Sivashinsky, Propagation of a pulsating reaction front in solid fuel combustion, SIAM J. Appl. Math., 35 (1978), pp. 465-478.

[15] A. G. Merzhanov, SHS processes: Combustion theory and practice, Arch. Combust., 1 (1981), pp. 23-48.

[16] A. G. Merzhanov, A. K. Filonenko, and I. P. Borovinskaya, New phenomena in combustion of condensed systems, Soviet Phys. Dokl., 208 (1973), pp. 892-894.

[17] Z. A. Munir and U. Anselmi-Tamburini, Self-propagating exothermic reactions: The synthesis of high-temperature materials by combustion, Mat. Sci. Rep., 3 (1989), pp. 277-365. 
[18] K. G. Shkadinsky, B. I. Khaikin, And A. G. Merzhanov, Propagation of a pulsating exothermic reaction front in the condensed phase, Combust. Expl. Shock Waves, 7 (1971), pp. 1522.

[19] G. I. Sivashinsky, The structure of Bunsen flames, J. Chem. Phys., 62 (1975), pp. 638-643.

[20] A. Varma, A. S. Rogachev, A. S. Mukasyan, and S. Huang, Combustion synthesis of advanced materials: Principles and applications, Adv. Chem. Engrg., 24 (1998), pp. 79226.

[21] J. YU AND L. K. GRoss, The onset of linear instabilities in a solid combustion model, Stud. Appl. Math., 107 (2001), pp. 81-101.

[22] J. YU AND Y. YANG, Evolution of small periodic disturbances into roll waves in channel flow with internal dissipation, Stud. Appl. Math., 111 (2003), pp. 1-27. 